\title{
Effects of acute consumption of a fruit and vegetable purée-based drink on vasodilation and oxidative status
}

\author{
Trevor W. George ${ }^{1}$, Saran Waroonphan ${ }^{1}$, Chutamat Niwat ${ }^{1}$, Michael H. Gordon ${ }^{1}$ \\ and Julie A. Lovegrove ${ }^{1,2 *}$ \\ ${ }^{1}$ Hugh Sinclair Unit of Human Nutrition, Department of Food and Nutritional Sciences, School of Chemistry, \\ Food and Pharmacy, University of Reading, Whiteknights, PO Box 226, Reading, Berks RG6 6AP, UK \\ ${ }^{2}$ Institute for Cardiovascular and Metabolic Research (ICMR), University of Reading, Whiteknights, PO Box 226, \\ Reading, Berks RG6 6AP, UK
}

(Submitted 3 November 2011 - Final revision received 19 June 2012 - Accepted 26 June 2012 - First published online 28 September 2012)

\begin{abstract}
Epidemiological studies indicate that diets rich in fruits and vegetables (F\&V) are protective against CVD. Puréed F\&V products retain many beneficial components, including flavonoids, carotenoids, vitamin $\mathrm{C}$ and dietary fibres. The present study aimed to establish the physiological effects of acute ingestion of a F\&V purée-based drink (FVPD) on vasodilation, antioxidant status, phytochemical bioavailability and other CVD risk factors. A total of twenty-four subjects, aged 30-70 years, completed the randomised, single-blind, controlled, crossover test meal study. Subjects consumed $400 \mathrm{ml}$ of the FVPD, or a fruit-flavoured sugar-matched control, after following a low-flavonoid diet for $5 \mathrm{~d}$. Blood and urine samples were collected throughout the study day, and vascular reactivity was assessed at 90 min intervals using laser Doppler iontophoresis. The FVPD significantly increased plasma vitamin $C(P=0.002)$ and total nitrate/nitrite $(P=0 \cdot 001)$ concentrations. There was a near significant time by treatment effect on ex vivo LDL oxidation $(P=0.068)$, with a longer lag phase after consuming the FVPD. During the $6 \mathrm{~h}$ after juice consumption, the antioxidant capacity of plasma increased significantly $(P=0.003)$ and there was a simultaneous increase in plasma and urinary phenolic metabolites $(P<0.05)$. There were significantly lower glucose and insulin peaks after ingestion of the FVPD compared with control $(P=0.019$ and 0.003$)$ and a trend towards increased endothelium-dependent vasodilation following FVPD consumption $(P=0 \cdot 061)$. Overall, FVPD consumption significantly increased plasma vitamin $\mathrm{C}$ and total nitrate/nitrite concentrations, with a trend towards increased endothelium-dependent vasodilation. Puréed F\&V products are useful vehicles for increasing micronutrient status, plasma antioxidant capacity and in vivo NO generation, which may contribute to CVD risk reduction.
\end{abstract}

Key words: Juice: Flavonoids: Vascular reactivity: CVD

Many epidemiological studies have shown that a high consumption of fruits and vegetables is associated with a reduced risk of CVD and other chronic diseases ${ }^{(1-3)}$. CVD is responsible for about half (48\%) of all deaths annually in Europe and is the major cause of death in men and women in the $\mathrm{UK}^{(4)}$. Endothelial dysfunction is a central feature in the early development of CVD, and impairment of endotheliumdependent vasodilation has been shown to precede structural atherosclerotic lesions ${ }^{(5)}$. The vascular endothelium displays systemic characteristics, which enables non-invasive measurements of peripheral circulation to be used as an indicator of the condition of the coronary arteries ${ }^{(6)}$. Laser Doppler imaging coupled with iontophoresis (LDI) is one such method of investigating the vasodilation of the peripheral microvasculature by delivering vasodilator agents across the skin under the influence of an applied electrical field ${ }^{(7)}$. Acetylcholine is administered at the anode and tests endothelial function via its vasodilator action of binding to muscarinic receptors on endothelial cells, subsequently generating $\mathrm{NO}$ via endothelial NO synthase (eNOS), thereby acting as an 'endotheliumdependent' vasodilator. A NO donor, sodium nitroprusside, is administered at the cathode and acts as an 'endotheliumindependent' vasodilator, which acts as a control to test the integrity of vascular smooth muscle. Many fruits, vegetables and their juices contain polyphenolic flavonoid compounds ${ }^{(8,9)}$ that have been shown to increase eNOS activity in cell and animal studies at physiological concentrations ${ }^{(10-15)}$. In addition, there is a growing body of evidence that supports the beneficial impact of chronic fruit and vegetable consumption ${ }^{(16-18)}$ and isolated flavonoids ${ }^{(19,20)}$ on

Abbreviations: eNOS, endothelial NO synthase; FRAP, ferric-reducing antioxidant power; FVPD, fruit and vegetable purée-based drink; LDI, laser Doppler iontophoresis.

*Corresponding author: J. A. Lovegrove, fax +44118931 0080, email j.a.lovegrove@reading.ac.uk 
improvements in measures of vascular function. However, the acute in vivo vascular response to fruit and vegetable consumption in human subjects is very limited $^{(21)}$ and has not been adequately assessed.

Oxidation of LDL has been recognised as an early stage in the development of atherosclerosis leading to $\mathrm{CVD}^{(22-25)}$. Many studies have reported the antioxidant effects of phytochemicals in fruits and vegetables, which include the retardation of the susceptibility of LDL to oxidation in vitro and in vivo ${ }^{(22-24,26)}$. Plant foods contain many components that could contribute to significant health benefits, including vitamins, fibre, carotenoids, sulphur compounds, nitrate and organic acids, as well as a wide variety of phenolic phytochemicals $^{(26-29)}$. Compounds in the latter class may contribute to benefits by reducing oxidative stress $^{(27)}$, by regulating enzymes involved in phase 2 metabolism, as well as by other mechanisms. The beneficial components listed earlier are also retained in many fruit and vegetable juices and purée products ${ }^{(30-32)}$. Research has shown that fruit and vegetable juices also have a protective effect against CVD risk ${ }^{(17,33)}$. In the UK, the average adult consumes less than the recommended minimum of five $80 \mathrm{~g}$ portions of fruits and vegetables per $\mathrm{d}^{(34)}$, but there has been a continued increase in fruit juice and liquidised/puréed product consumption in the $\mathrm{UK}^{(35)}$, and these products represent an important source of micronutrients. The aim of the present study was to investigate the acute effects of the consumption of a concentrated fruit and vegetable purée-based drink on vascular function, antioxidant status and plasma concentrations of beneficial phytochemicals and vitamin $\mathrm{C}$, which could contribute to a reduction in the risk of chronic diseases, such as CVD.

\section{Experimental methods}

\section{Study population}

A total of twenty-four subjects (twenty males and four females), aged between 30 and 70 years, completed the study. The subjects were recruited from the University of Reading and general public around the Reading area, and were selected if they met the study criteria of no known liver disease, diabetes mellitus or a diagnosed mycocardial infarction; no gall bladder problems or abnormalities of fat metabolism; no subjects on weight-reducing diets or taking dietary supplements; no vigorous exercise or excess consumption of alcohol; BMI $<30 \mathrm{~kg} / \mathrm{m}^{2}$; blood pressure $<150 / 90 \mathrm{mmHg}$ and $\mathrm{Hb}>125 \mathrm{~g} / \mathrm{l}$. All study subjects had basic anthropometric measurements recorded, and fasting blood was taken and analysed for liver function status, lipid levels, fasting glucose and a measure of potential alcohol abuse. A total of twenty-five subjects were required to be recruited to detect a $10 \%$ difference in acetylcholine vasodilatory response measured by LDI based on the present data for intra-subject reproducibility response (within-day and between-day variation, both $12 \%$ ), with $P<0.05,80 \%$ power and an allowance for a $10 \%$ drop-out. The present study was conducted according to the guidelines laid down in the Declaration of Helsinki, and all procedures involving human subjects were assessed by the University of Reading Research Ethics Committee who gave a favourable ethical opinion for conduct. Each subject gave written informed consent before participating. The registration number for clinical trials is ISRCTN36287115.

\section{Study design}

The study was a randomised, single-blind, controlled, crossover, acute postprandial test meal study. The subjects were randomly assigned to either the fruit and vegetable puréebased drink (FVPD) or the control drink. The subjects consumed a low-flavonoid diet for a $5 \mathrm{~d}$ period preceding the study day. On the study day, a flexible cannula was inserted into the forearm and blood samples taken at baseline and at twelve additional time points after consumption of the relevant drink, eight samples $30 \mathrm{~min}$ apart followed by four samples $1 \mathrm{~h}$ apart. Urine was collected before the drink was consumed and then at $2 \mathrm{~h}$ intervals for $8 \mathrm{~h}$ of the study day. LDI was used to record a real-time measure of vascular reactivity for two baseline measurements and five measurements following drink consumption, all at $90 \mathrm{~min}$ intervals. The whole procedure was repeated with the other intervention drink after a 4-week washout.

\section{Intervention drinks}

Subjects were asked to consume $400 \mathrm{ml}$ FVPD (Vie Shots; Unilever Bestfoods) or $400 \mathrm{ml}$ fruit-flavoured cordial (Robinsons Lemon Barley Water; Robinsons Limited), which was matched for sugar composition and diluted with low-nitrate mineral water (The Buxton Mineral Water Company Limited). The FVPD was a fruit and vegetable drink made from $800 \mathrm{~g}$ fruit and vegetables in the form of concentrated juices and purées, which was composed of apple (56\%), carrot (29\%) and strawberry $(8 \%)$. The nutrient composition of the drinks is shown in Table 1.

\section{Fruit and vegetable purée-based drink antioxidant components}

FVPD extracts were prepared by extraction with acidified methanol ${ }^{(36)}$. Total phenolic compounds were determined by the Folin-Ciocalteu method ${ }^{(37)}$, and flavonoid content and composition were determined by HPLC ${ }^{(24)}$. The polyphenol content of the FVPD was determined by the method of Garcia-Macias et $a l^{(38)}$. Aqueous antioxidant activity was assessed by the oxygen radical absorbance capacity method ${ }^{(39)}$. A volume of FVPD extract containing $0.5 \mu \mathrm{m}$-gallic acid equivalents, determined by the FolinCiocalteu method, was incubated with isolated LDL containing $100 \mu \mathrm{g}$ protein in the assay.

\section{Anthropometric measurements}

Measurements of height, weight, BMI and blood pressure were recorded at the start of each of the two study days. 
Table 1. Nutrient composition of fruit and vegetable purée-based drink (FVPD) and control drink*

\begin{tabular}{lcc}
\hline & FVPD & Control \\
& $(400 \mathrm{ml})$ & $(400 \mathrm{ml})$ \\
\hline Energy & & \\
kcal & 252 & 203 \\
kJ & 1054 & 849 \\
Protein (g) & $4 \cdot 0$ & $0 \cdot 3$ \\
Carbohydrate (g) & $54 \cdot 4$ & $50 \cdot 4$ \\
Of which sugar (g) & $50 \cdot 4$ & $50 \cdot 4$ \\
Fat (g) & $2 \cdot 0$ & Trace \\
Of which saturates (g) & 0.4 & Trace \\
Fibre (g) & $6 \cdot 0$ & - \\
Na (g) & $0 \cdot 12$ & - \\
Ascorbic acid (mg) & 120 & - \\
Nitrate/nitrite (mg) & $1 \cdot 0$ & $0 \cdot 0$ \\
Total carotenoids (mg) & $20 \cdot 4$ & - \\
Total phenolics (mg GAE) & 768 & 44 \\
Caffeic acid (mg) & 60 & - \\
Ferulic acid (mg) & 16 & - \\
Epicatechin (mg) & 457 & - \\
Chlorogenic acid (mg) & 31 & - \\
Cyanidin (mg) & 53 & - \\
Pelargonidin-3-glucoside (mg) & 37 & - \\
Pelargonidin (mg) & 11 & - \\
\hline
\end{tabular}

GAE, gallic acid equivalents.

${ }^{*}$ Adapted from George et al. ${ }^{(65)}$

\section{Plasma collection and analysis}

Blood samples were collected via syringe from a cannula and transferred to citrate, EDTA and lithium heparin vacutainer tubes. The tubes were immediately wrapped in foil and kept on ice for transport to the laboratory. Following centrifugation at $4^{\circ} \mathrm{C}$ at $3000 \mathrm{rpm}$, plasma was aliquotted into separate cryogenic vials for storage at $-80^{\circ} \mathrm{C}$. Analysis did not commence until the intervention study was completed, and all samples from each subject were analysed within one batch to reduce inter-batch variation.

Analyses of plasma TAG, total and HDL-cholesterol, NEFA and glucose were performed using an Instrument Laboratory ILAB 600 autoanalyser and standard kits (Instrumental Laboratories Limited). Appropriate sero-normal, low and high quality control standards (Instrument Laboratories Limited) were included in all batches. Insulin was assessed by ELISA (Dako Cytomation; Ely) with in-house pooled plasma controls in each batch.

Plasma samples for ascorbic and uric acid analysis were treated with 5\% metaphosphoric acid (1:1, v/v) and stored at $-80^{\circ} \mathrm{C}$ prior to analysis by HPLC with UV detection ${ }^{(40,41)}$. Total nitrate/nitrite was measured using an ELISA kit (Active Motif; Rixensart) based on the Greiss reaction ${ }^{(42)}$ in plasma, FVPD and the control drink.

The ferric-reducing antioxidant power (FRAP) of plasma was determined using the method of Benzie \& Strain ${ }^{(43)}$, adapted for use with ninety-six-well microtitre plates ${ }^{(44)}$. A Genios spectrophotometer (Tecan Limited) was used to measure the absorbance at $593 \mathrm{~nm}$. This method was used to estimate aqueous antioxidants. The method for LDL isolation and assessment of oxidative stability was based on that of Leigh-Firbank et $a l .{ }^{(45)}$. Concentrations of $50 \mu \mathrm{g}$ of LDL protein $/ \mathrm{ml}$ and $5 \mu \mathrm{M}-\mathrm{CuSO}_{4}$ were used for oxidation at $\mathrm{pH}$
7.4. The formation of conjugated dienes (the breakdown products of lipid peroxidation) was monitored at $234 \mathrm{~nm}$, $37^{\circ} \mathrm{C}$ every $2 \mathrm{~min}$ for $3 \mathrm{~h}$ using a Perkin-Elmer Lambda bio 20 UV/VIS Spectrometer ${ }^{(46)}$.

Plasma $(300 \mu \mathrm{l})$ was extracted four times with $0.5 \mathrm{ml}$ of ethyl acetate by vortexing for $1 \mathrm{~min}$ after addition of $100 \mu \mathrm{l}$ of $5 \mathrm{~m}-\mathrm{HCl}$. The mixture was then centrifuged at $13200 \mathrm{rpm}$ for $10 \mathrm{~min}$, and the top layer removed. The ethyl acetate extracts were dried and derivatised by the addition of $200 \mu \mathrm{l}$ of $N, O$-bis(trimethylsilyl) trifluoroacetamide + trimethylchlorosilane and heated at $70^{\circ} \mathrm{C}$ for $4 \mathrm{~h}^{(47)}$. An Agilent Technologies Model GC $6890 \mathrm{~N}$ (G1530N) gas chromatograph coupled with an Agilent 5975 series Inert XL mass selective detector and a CTC analysis system autosampler were used for analyses. Samples $(2.0 \mu \mathrm{l})$ were analysed on a $30 \mathrm{~m}, 0.25 \mathrm{~mm}$ inner diameter, $0.50 \mu \mathrm{m}$, DB-5-fused silica capillary column (Agilent Technologies), with temperature programming from $80^{\circ} \mathrm{C}$ ( $1 \mathrm{~min}$ ) increasing up to $220^{\circ} \mathrm{C}$ at a rate of $10^{\circ} \mathrm{C} / \mathrm{min}$ and from 220 to $310^{\circ} \mathrm{C}$ at a rate of $10^{\circ} \mathrm{C} / \mathrm{min}$ and held for $6 \mathrm{~min}$. Electron-impact mass spectra were recorded with ionisation energy of $70 \mathrm{eV}$. o-Phthalic acid was used as an internal standard.

\section{Urinary creatinine, hippuric and phenolic acids}

Analysis of urinary creatinine was performed using an Instrument Laboratory ILAB 600 autoanalyser. Urinary hippuric acid was measured as an indicator of polyphenol metabolism ${ }^{(48)}$ and determined by the extraction method of Mohsen et al. ${ }^{(49)}$ and analysed by HPLC by adapting the methods of Felgines et $a{ }^{(50)}$ and Kay et $a{ }^{(51)}$.

\section{Laser Doppler imaging with iontophoresis}

Subjects were in a supine position, in a quiet room at an ambient temperature of $22 \pm 1^{\circ} \mathrm{C}$ for all measures. Two ION6 Perspex chambers (Moor Instruments Limited) with an internal platinum wire electrode were placed on the volar aspect of the forearm and attached to the skin using adhesive discs (MIC-1AD; Moor Instruments Limited) and connected to a MIC2 iontophoresis controller (Moor Instruments Limited). Skin temperature was recorded at the time of measurement. Acetylcholine chloride $(2.5 \mathrm{ml}, 1 \%$; Sigma-Aldrich) in $0.5 \%$ $\mathrm{NaCl}$ solution was placed in the anodal chamber and $2.5 \mathrm{ml}$ of $1 \%$ sodium nitroprusside (Sigma-Aldrich) in $0.5 \% \mathrm{NaCl}$ solution was placed in the cathodal chamber. Circular glass coverslips were placed over each chamber to prevent loss of solutions. Current delivery was controlled by laser Doppler imager Windows software 5.1 (Moor Instruments Limited). Measurement of skin perfusion was carried out using a moorLDI2-IR laser Doppler imager (Moor Instruments Limited). The scanner head was positioned $30 \mathrm{~cm}$ above the chambers. The laser light was directed by a moving mirror in a raster fashion over both chambers. A total of ten repeat scans were taken, the first with no current to act as a control, then two scans at $10 \mu \mathrm{A}$, two at $20 \mu \mathrm{A}$, two at $30 \mu \mathrm{A}$ and one at $40 \mu \mathrm{A}$ to give a total charge of $8 \mathrm{mC}$, the final two scans were measured without any current. The area under the flux 
Table 2. Baseline characteristics of subjects in acute fruit and vegetable purée-based drink consumption study

(Mean values and standard deviations)

\begin{tabular}{lccccc}
\hline & \multicolumn{2}{c}{ Males $(n$ 20) } & & \multicolumn{2}{c}{ Females $(n$ 4) } \\
\cline { 2 - 3 } & Mean & SD & & Mean & SD \\
\hline Age (years) & 46 & 11 & & 49 & 4 \\
Weight $(\mathrm{kg})$ & 79.9 & 10.4 & & $66 \cdot 7$ & 7.9 \\
BMI $\left(\mathrm{kg} / \mathrm{m}^{2}\right)$ & 25.6 & 3.2 & & 24.0 & 2.6 \\
Systolic BP $(\mathrm{mmHg})$ & 125 & 12 & & 106 & 12 \\
Diastolic BP $(\mathrm{mmHg})$ & 79 & 7 & & 71 & 7 \\
Glucose $(\mathrm{mmol} / \mathrm{l})$ & 5.0 & 0.3 & & 4.9 & 0.1 \\
Total cholesterol $(\mathrm{mmol} / \mathrm{l})$ & 5.3 & 0.9 & & 4.5 & 0.8 \\
TAG (mmol/l) & 1.4 & 0.9 & & 0.8 & 0.2 \\
\hline
\end{tabular}

$\mathrm{BP}$, blood pressure.

$v$. time curve over the ten scans was deemed to indicate the microvascular response.

\section{Statistical analyses}

All statistical analyses were performed using SPSS 13.0 for Microsoft Windows (SPSS Inc.). The data were checked for normality using the Shapiro-Wilk test, as the number of subjects was less than fifty. Those data that were not normallydistributed were log transformed and reassessed. A repeatedmeasures ANOVA was used to detect significant differences between treatment groups with Bonferroni correction to reduce the likelihood of chance findings from multiple comparisons. A value of $P \leq 0.050$ was used to define significance and a $95 \%$ CI. The data presented in tables and graphs are displayed as means with their standard errors, unless otherwise stated.

\section{Results}

Antioxidant components of fruit and vegetable puréebased drink

The antioxidant components of the FVPD are listed in Table 1. The FVPD contained a high concentration of flavonoids, with $400 \mathrm{ml}$ of the FVPD supplying $768 \mathrm{mg}$ of total phenolics (gallic acid equivalents) including $100 \mathrm{mg}$ of anthocyanins. The control drink contained only $44 \mathrm{mg}$ of total phenolics (gallic acid equivalents) with no anthocyanins. The FVPD had a high antioxidant capacity when assessed by the oxygen radical absorbance capacity assay (6702 $\mu \mathrm{m}$ Trolox equivalents).

\section{Anthropometric data}

The baseline anthropometric data for the subjects are shown in Table 2. There was no change in weight, BMI or blood pressure between study days (data not shown).

\section{Biochemical data}

There was a significant time by treatment effect $(P<0 \cdot 001)$ and a significant increase in plasma ascorbic acid concentration following FVPD consumption at all time points from $60 \mathrm{~min}$

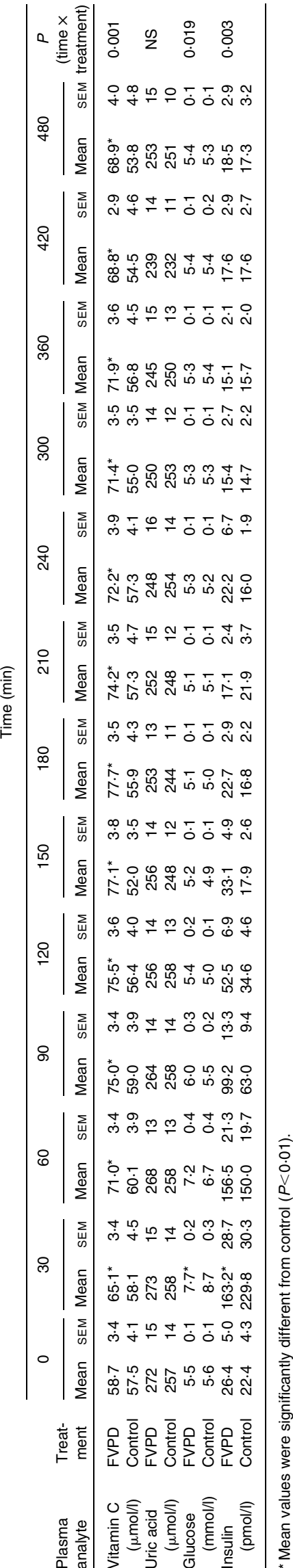




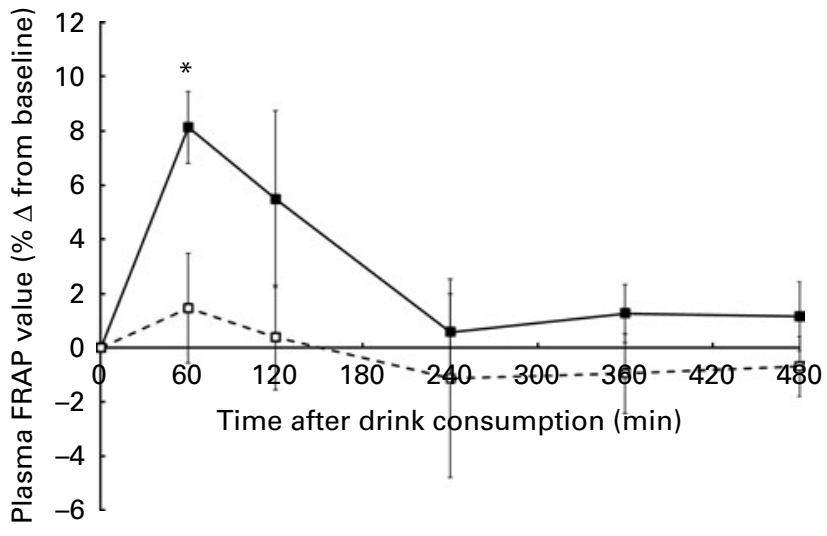

Fig. 1. Ferric-reducing antioxidant potential (FRAP) of plasma (percentage change from baseline) following acute consumption of fruit and vegetable purée-based drink (FVPD) or control $(n$ 24). The solid line represents the effect from FVPD consumption and the dashed line represents the effect from the control. There was a significant time by treatment effect $(P=0.03)$. * Mean values were significantly different for effect between treatments after post hoc tests.

after ingestion, reaching a maximum value approximately $120 \mathrm{~min}$ after consumption. The plasma uric acid concentration did not change significantly (Table 3). There was a significant increase in oxidative stability determined by the FRAP assay after FVPD consumption, as shown in Fig. 1 (time by treatment, $P=0 \cdot 030)$. The peak FRAP response occurred $60 \mathrm{~min}$ after FVPD consumption $(P=0 \cdot 003)$. It was observed that there was a near significant attenuation in the reduction in the postprandial LDL oxidation lag phase response (time by treatment, $P=0.068$ ) after the FVPD compared with the control drink, with a significant difference in LDL oxidation lag phase between treatments at $60 \mathrm{~min}$ after FVPD consumption $(P=0.016$; Fig. 2$)$. There was no correlation between the concentration of either plasma vitamin $\mathrm{C}$ or uric acid with plasma antioxidant capacity assessed by the FRAP and LDL oxidation assays (data not shown).

There was a significant time by treatment interaction $(P=0.03)$ in the plasma concentration of salicylic acid, with a significant increase from 1 to $5 \mathrm{~h}$ after FVPD consumption (Table 4, $P<0 \cdot 0001)$. Plasma benzoic acid concentration tended to increase at $2 \mathrm{~h}$ after FVPD consumption and almost reached statistical significance at $6 \mathrm{~h}$ (Table 4 , $P=0.07)$. The benzoic acid concentration in the FVPD group remained higher than observed after consumption of the control drink throughout the intervention period. Plasma p-hydroxyphenylacetic acid and 3-(4-hydroxy phenyl)-2hydroxypropanoic acid concentrations remained higher than the response to the control drink throughout the $8 \mathrm{~h}$ intervention period (Table 4). Both compounds peaked $90 \mathrm{~min}$ after FVPD consumption, but only $p$-hydroxyphenylacetic acid reached a maximum that was significantly higher than the control (Table $4, P=0 \cdot 01$ ). After consuming the FVPD, the urinary concentration of vanillic acid, $p$-hydroxybenzoic acid and ferulic acid remained higher than the control treatment throughout the intervention period (Fig. 3), the mean peak concentration occurred after $4 \mathrm{~h}$ for vanillic and $p$-hydroxybenzoic acid $(P<0.001$ and 0.04 , respectively) as shown in Fig. 3(a) and (b). Ferulic acid was only found in urine after subjects consumed the FVPD, and the concentration peaked after $2 \mathrm{~h}$ and remained constant until $6 \mathrm{~h}$ before reducing. There was a highly significant time by treatment effect $(P<0.0001)$ in the plasma concentration of hippuric acid, with a significant increase from 3 to $8 \mathrm{~h}$ after FVPD consumption only (Table 4 ). There was also a significant time by treatment effect $(P=0.008)$ in the urinary excretion of hippuric acid, with a significant increase at 360 and $480 \mathrm{~min}$ after FVPD consumption $(P=0.0001$ and $0 \cdot 010$, respectively) as shown in Fig. 3(d).

A significant time by treatment effect was observed for the glucose and insulin response (Table 3, $P=0.019$ and 0.003, respectively), with a significantly lower peak concentration after consumption of the FVPD compared to the sugarmatched control $(P=0.004$ and 0.029 , respectively). There was no effect of FVPD consumption on plasma TAG, total or HDL-cholesterol or NEFA (data not shown). There was a significant time by treatment interaction $(P<0.001)$ and a significant increase in total plasma nitrate/nitrite concentration following FVPD consumption at all time points after ingestion (Fig. 4).

\section{Laser Doppler imaging with iontophoresis}

The endothelium-dependent vasodilation response to acetylcholine is shown in Fig. 5. There was a trend towards increased vasodilation following FVPD consumption throughout the day, which almost reached statistical significance $(P=0 \cdot 061)$

\section{Discussion}

The present study is the first to investigate the effects of an acute ingestion of fruits and vegetables, in the form of $400 \mathrm{ml}$ of a purée-based drink, on vascular reactivity and plasma and urine phytochemical composition. The FVPD contained significant levels of vitamin $\mathrm{C}$ and polyphenols

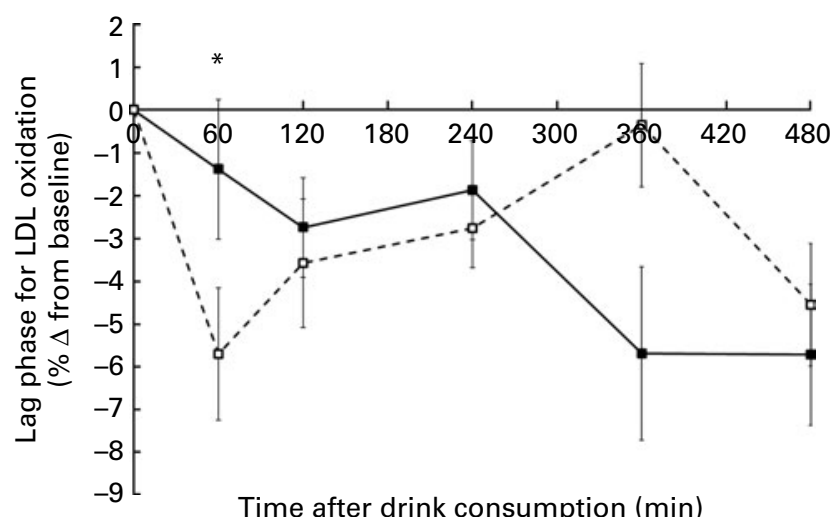

Fig. 2. LDL lag phase time (percentage change from baseline) following acute consumption of fruit and vegetable purée-based drink (FVPD) or control $(n 24)$. The solid line represents the effect from FVPD consumption and the dashed line represents the effect from the control. There was a near significant time by treatment effect $(P=0.068)$. ${ }^{*}$ Mean values were significantly different for effect between treatments after post hoc tests. 
from the fruit sources, including anthocyanins, other flavonoids and phenolic acids. The present study is one of a few studies that have investigated the effect of an acute ingestion of polyphenol-rich drink on postprandial LDL oxidation. A major finding of the present study was that the susceptibility of LDL to oxidation increased throughout the $8 \mathrm{~h}$ of the intervention period after subjects fasted overnight and consumed either the FVPD or control. A fall in the lag phase time in subjects who had fasted for at least $12 \mathrm{~h}$ was also reported by Hodgson et $\mathrm{al}^{\left({ }^{(52)}\right.}$, who found that LDL isolated at $60 \mathrm{~min}$ after consumption of water or water-containing caffeine had lag phases that were shorter than the baseline values. However, the acute consumption of the FVPD significantly increased the lag phase compared to control after $60 \mathrm{~min}$ for all subjects $(P=0.016)$ and the lag phase remained longer than control over a $4 \mathrm{~h}$ period. The increase in lag phase cannot be due to carotenoid components, because most of the increase in plasma carotenoids occurs after more than $2 \mathrm{~h}^{(53)}$. There was a significant increase in plasma ascorbic acid following FVPD consumption, with a maximum concentration reached at $150-180 \mathrm{~min}$, indicating that the ascorbic acid in the FVPD was effectively absorbed. An increase in plasma oxidative stability, assessed by the FRAP assay from 60 to $180 \mathrm{~min}$, suggested that the antioxidant components of the FVPD, including ascorbic acid and flavonoids, increased the antioxidant capacity. There was no effect of treatment on plasma uric acid, which was not surprising as the control drink was matched for sugar composition, which was necessary as previously it has been shown that fructose increases plasma uric acid ${ }^{(54)}$. Caccetta et al. ${ }^{(55)}$ found a significant increase in plasma uric acid over $4 \mathrm{~h}$, with a peak at $60 \mathrm{~min}$ after the subjects consumed $350 \mathrm{ml}$ of red wine, but wine consumption had no effect on LDL oxidation.

In the present study hippuric acid was the main flavonoid metabolite found in plasma and urine. The result was comparable to previously reported studies, after consumption of phenolic compounds such as flavanols, quercetin or chlorogenic acid ${ }^{(26,48,56,57)}$. When these compounds reach the colon, they can be converted to valerolactone and then to phenolic acids by the action of gut microflora, which are then converted to benzoic acid by $\beta$ oxidation in the liver and benzoic acid is conjugated with glycine to form $N$-benzoylglycine or hippuric acid, which can be absorbed or excreted in urine $^{(48,56)}$. Valentova et al. ${ }^{(57)}$ observed that after subjects consumed $1200 \mathrm{mg}$ of dried cranberry juice/d for 8 weeks (estimated daily intake of $35 \mathrm{mg}$ total phenolics), the major metabolite found in the urine was hippuric acid, which was present at a significantly higher concentration than the control. Additionally, the serum levels of advanced protein oxidation products were decreased at the end of the intervention. However, in contrast, no correlation between plasma/urinary hippuric acid and plasma antioxidant status was observed in the present study (data not shown). Hippuric acid has no antioxidant activity, due to the absence of a phenolic hydroxyl group in the molecule. In support of this, no correlation was observed between flavonoid metabolites in plasma and urine with antioxidant status after consumption of the FVPD. Plasma antioxidant status assessed by LDL oxidation and by 
(a)
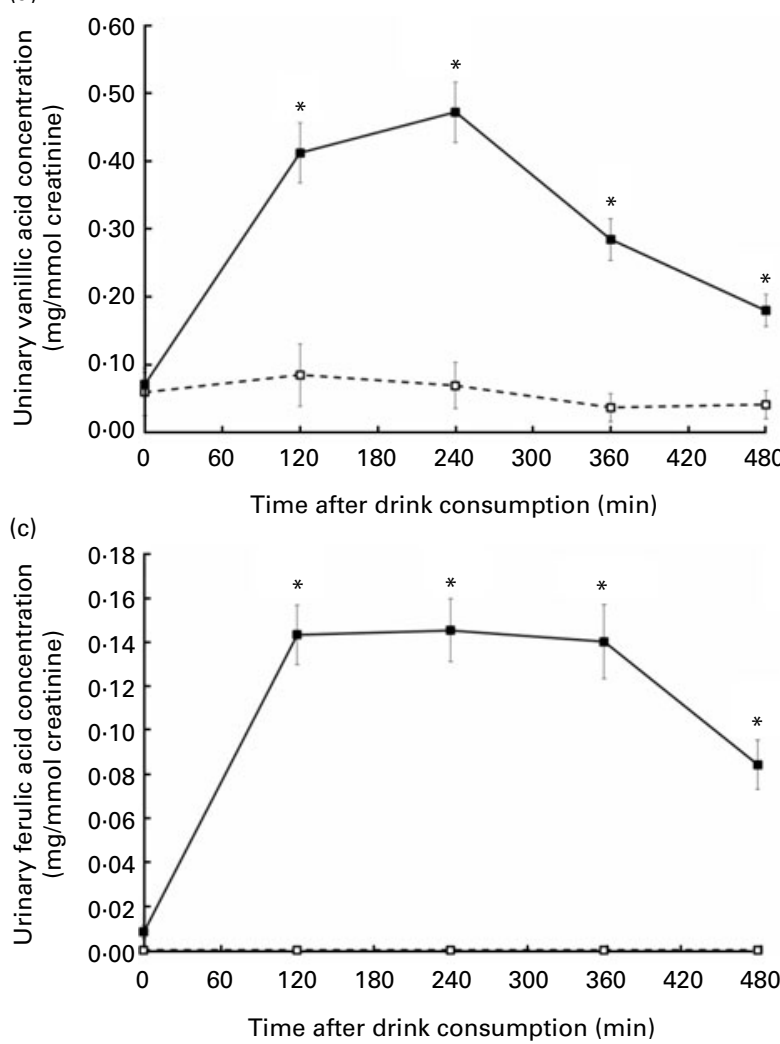

(b)

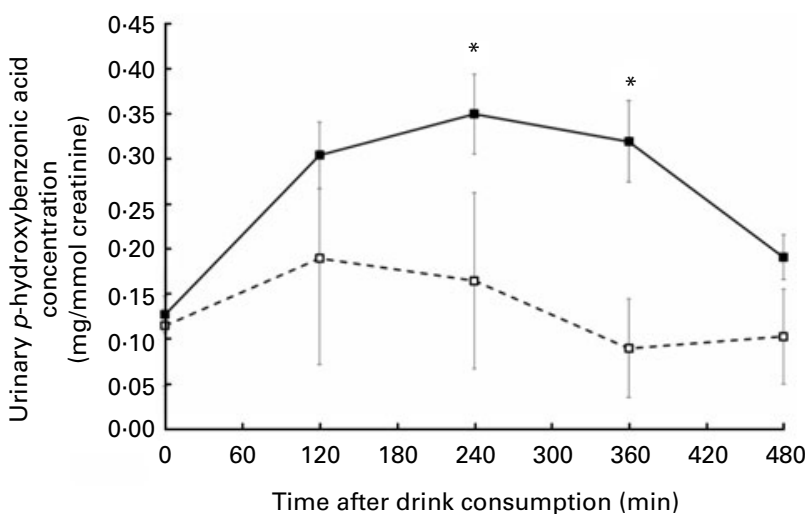

(d)

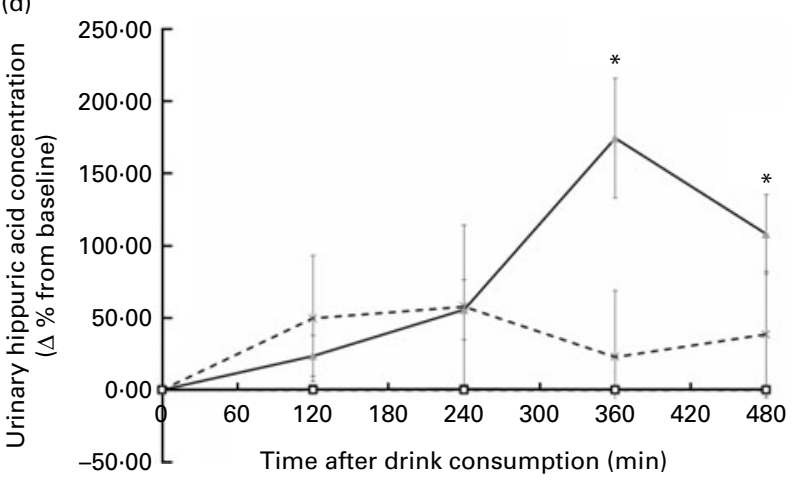

Fig. 3. Urinary phenolic acid ((a) vanillic acid; (b) p-hydroxybenzoic acid; and (c) ferulic acid) as $\mathrm{mg} / \mathrm{mmol}$ creatinine, and (d) hippuric acid as \% change from baseline following acute consumption of fruit and vegetable purée-based drink (FVPD) or control $(n 24)$. The solid lines represent the effect from FVPD consumption and the dashed lines represent the effect from the control. The mean peak concentration occurred after $4 \mathrm{~h}$ for vanillic and $p$-hydroxybenzoic acid $(P<0.001$ and 0.04 , respectively). Ferulic acid was only found in urine after subjects consumed the FVPD, and the concentration peaked after $2 \mathrm{~h}$ and remained constant until $6 \mathrm{~h}$ before reducing (time by treatment, $P<0.001$ ). There was a highly significant time by treatment effect in urinary hippuric acid $(P<0.008) .{ }^{*}$ Mean values were significantly different for effect between treatments after post hoc tests.

the FRAP assay peaked at 60 min after consumption of the FVPD. The increase in oxidative stability at $60 \mathrm{~min}$ is expected to be mainly due to the protection of LDL by the increased plasma vitamin $\mathrm{C}$ concentration prior to isolation of the LDL. Hippuric acid reaching a peak at 5 and $6 \mathrm{~h}$ in plasma and urine, respectively. The delayed appearance of hippuric acid reflected the fact that this compound was formed in the colon due to bacterial metabolism and subsequently absorbed and excreted in the plasma and urine, respectively ${ }^{(26,48,56,57)}$. Epicatechin was the major flavonoid detected in the FVPD, followed by anthocyanins and caffeic acid (Table 1). However, apart from hippuric acid, which is the major metabolite of flavanols and hydroxycinnamates, other metabolites such as glucuronides, sulphates and $O$-methylates, in addition to anthocyanins, were not detected in the plasma or urine samples. The major metabolites detected were phenolic acids. These are secondary metabolites derived from the biotransformation of colonic microflora. The method used to analyse plasma was relatively insensitive in detecting intact flavonoid derivatives.

Several studies have investigated the effect of acute consumption of foods on Cu-catalysed LDL oxidation. Natella et $a l .{ }^{(58)}$ reported that acute consumption of a cup of coffee significantly reduced the susceptibility of LDL to oxidation by increasing the lag phase of LDL isolated from blood removed at 30 and $60 \mathrm{~min}$ after consumption. Hodgson et $a l .{ }^{(52)}$ found no significant effect of the acute consumption of green tea, black tea and water containing $180 \mathrm{mg}$ caffeine on LDL oxidation lag phase and total antioxidant capacity of

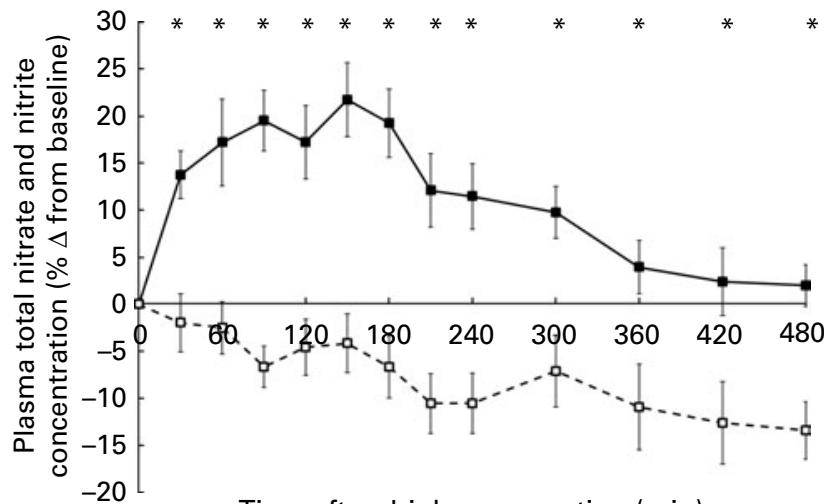

Time after drink consumption ( $\mathrm{min}$ )

Fig. 4. Total plasma nitrate/nitrite concentration (\% change from baseline) following acute consumption of fruit and vegetable purée-based drink (FVPD) or control $(n 24)$. The solid line represents the values after FVPD consumption and the dashed line represents the values for the control. There was a significant time by treatment effect $(P=0.001)$. * Mean values were significantly different for effect between treatments after post hoc tests. 


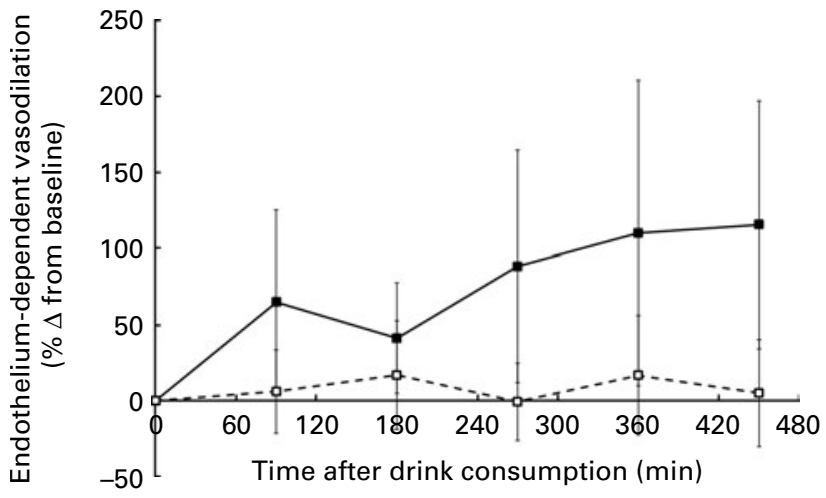

Fig. 5. Endothelium-dependent vasodilation in response to acetylcholine (percentage change from baseline) following acute consumption of fruit and vegetable purée-based drink (FVPD) or control (n 24). The solid line represents the values after FVPD consumption and the dashed lines represent the values for the control.

samples isolated $60 \mathrm{~min}$ after consumption. Miyagi et al. ${ }^{(59)}$ reported that acute consumption of red wine containing $433 \mathrm{mg}$ of total phenolics caused a significant increase in the lag phase time of samples isolated 1 and $2 \mathrm{~h}$ after consumption. However, consumption of red grape juice containing $511 \mathrm{mg}$ of total phenolics had no effect. Caccetta et al. ${ }^{(55)}$ reported findings that contradict the latter study, with no effect on the susceptibility of LDL to oxidation after acute consumption of red wine containing $700 \mathrm{mg}$ of total polyphenols. Another postprandial study investigated the effect of consuming olive oil containing $2 \cdot 7,164$ and $366 \mathrm{mg} / \mathrm{kg}$ of phenolic compounds on LDL oxidation. The results showed that only the consumption of $366 \mathrm{mg} / \mathrm{kg}$ olive oil had an effect, retarding LDL oxidation by 9.5 and $15.2 \%$ in LDL isolated from blood removed 2 and $6 \mathrm{~h}$, respectively, after consumption of olive oil ${ }^{(60)}$. Consequently, the effect of polyphenol-rich foods on the susceptibility of LDL to oxidation in acute interventions depends on the nature and amount of polyphenols in the food, as well as on other variables. The fall in oxidative stability of LDL isolated from both test and control groups after baseline may be due to the effects of fasting. However, absorption or formation of flavonoid metabolites in the plasma of subjects may be the reason for the increase in plasma antioxidant capacity due to the FVPD.

The present study also found that there was a significantly lower glucose and insulin peak concentration after consumption of the FVPD compared to the sugar-matched control. As the FVPD contained a mixture of purées and concentrated juices, this may have been due to the matrices of the fruit and vegetable particles slowing down the absorption of the sugars, whereas the sugars in the control drink were dissolved in mineral water. This highlights an additional benefit to puréed fruits and vegetables over juices that contain minimal food matrix components. There was a significant increase in total plasma nitrate and nitrite following FVPD consumption. The control drink was specifically diluted with Buxton mineral water that had the lowest nitrate content of all commercially available mineral waters. This was chosen to reduce any confounding effects of nitrate in the control, affecting vasodilation measured by LDI. Although the FVPD contained a minimal concentration of nitrate/nitrite from the fruits and vegetables $(1.0 \mathrm{mg}$ in $400 \mathrm{ml}$ ), the plasma concentrations of nitrate/nitrite at each time point after consumption of the FVPD were significantly higher than the expected concentration provided by the drink. The increase in plasma nitrate/nitrite was maintained throughout the day, whereas research has shown that the plasma nitrate concentration from the ingestion of dietary sources reaches a peak after approximately $60 \mathrm{~min}$ and then declines ${ }^{(28,61)}$. Therefore, the increase in nitrate/nitrite following FVPD consumption could be due to flavonoid components within the drink increasing eNOS activity. In vitro studies have shown that flavonoids $^{(10-12)}$ at physiological concentrations ${ }^{(13)}$ can increase eNOS gene expression. The FVPD was particularly rich in (-)-epicatechin $(457 \mathrm{mg} / 400 \mathrm{ml})$ and this compound has been shown to increase eNOS activity ${ }^{(14,15)}$ and endothelium-1 release ${ }^{(15)}$. In support of these findings, acute consumption of epicatechin-rich apples and grapes (consumed as champagne) have been associated with beneficial effects on postprandial NO status and on in vivo vasodilation measured by $\mathrm{LDI}^{(62)}$ and flow-mediated dilation ${ }^{(21)}$ in randomised control human studies. In addition, increased plasma NO and increased vasodilation have been reported after chronic consumption of fruits and vegetables, which may be due in part to flavonoids and/or other bioactive components ${ }^{(16-18)}$ The significant increase in urinary hippuric acid at 360 and $480 \mathrm{~min}$ indicated that the flavonoid components of the FVPD were absorbed with metabolism into hippuric acid, which is a metabolite of several polyphenol compounds ${ }^{(48,56)}$.

The novel LDI results were encouraging. There was a trend towards increased endothelium-dependent vasodilation following ingestion of the FVPD compared to the control drink. The initial increase could be due to flavonoid components present in the FVPD increasing eNOS activity by scavenging superoxide ${ }^{(14)}$, which may explain the increase in total plasma nitrate and nitrite found after FVPD consumption. The largest increase in vasodilation was observed at approximately $7.5 \mathrm{~h}$ after FVPD consumption, which could be attributed, at least in part, to methylated flavonoid metabolites from the small intestine ${ }^{(63)}$. These metabolites have been shown to have an even greater ability to increase eNOS activity compared with the parent molecules by inhibiting endothelial NADPH oxidase, thereby reducing superoxide production $^{(64)}$. There was no effect of the FVPD on plasma TAG, total and HDL-cholesterol or NEFA, but this was not unexpected as no fat meal had been given with the drinks.

Overall, the present study provides evidence that consumption of fruits and vegetables in the form of purée-based drinks acutely increases plasma ascorbic acid, total plasma nitrate and nitrite concentrations, oxidative stability of plasma assessed by the FRAP and ex vivo Cu-catalysed LDL oxidation. Increased concentrations of phenolic metabolites were detected in plasma and urine after consumption of the FVPD, and it was concluded that the increase in the oxidative stability of LDL and antioxidant capacity of plasma could be due to components in the FVPD such as phenolic acids, flavonoids and their metabolites. There was a trend towards 
increased endothelium-dependent vasodilation following FVPD consumption, which may be due to increased eNOS activity or increased NO sparing indicated by the increased plasma nitrate and nitrite. Purée-based fruit and vegetable drinks are suitable vehicles for micronutrient and phytochemical ingestion, which may contribute to CVD risk reduction.

\section{Acknowledgements}

The authors acknowledge funding from the University of Reading Research Endowment Trust Fund; the Royal Thai Government (Ministry of Science and Technology) and Chiangmai University, Thailand; the Food Standards Agency, UK and Unilever Bestfoods (Germany). The authors would like to thank the study participants for their involvement in the study, Jan Luff for her help with recruitment and Dr Anna Macready for her assistance in manuscript formatting. The authors have no conflict of interest. T. W. G. and J. A. L. prepared the manuscript. T. W. G., J. A. L. and M. H. G. designed the study. T. W. G. and C. N. conducted the study. T. W. G., S. W. and C. N. performed the analysis. J. A. L. had primary responsibility for the final content. All authors read and approved the final manuscript. Preliminary findings from the present study were presented at a Postgraduate Symposium during the Summer Meeting of the Nutrition Society 2008 $8^{(65)}$ and at the International Symposium II on Human Health Effects of Fruit and Vegetables: FAVHEALTH $2007^{(66)}$.

\section{References}

1. Zhang X, Shu X, Xiang Y, et al. (2011) Cruciferous vegetable consumption is associated with a reduced risk of total and cardiovascular disease mortality. Am J Clin Nutr 94, 240-246.

2. Crowe FL, Roddam AW, Key TJ, et al. (2011) Fruit and vegetable intake and mortality from ischaemic heart disease: results from the European Prospective Investigation into Cancer and Nutrition (EPIC)-Heart study. Eur Heart J 32, 1235-1243.

3. van Duijnhoven FJB, Bueno-De-Mesquita HB, Ferrari P, et al. (2009) Fruit, vegetables, and colorectal cancer risk: the European Prospective Investigation into Cancer and Nutrition. Am J Clin Nutr 89, 1441-1452.

4. Allender S, Scarborough P \& Peto V (2008) Cardiovascular Disease Statistics. London: European Heart Network.

5. McLenachan JM, Williams JK, Fish RD, et al. (1991) Loss of flow-mediated endothelium-dependent dilation occurs early in the development of atherosclerosis. Circulation 84, 1273-1278.

6. Lieberman EH, Gerhard MD, Uehata A, et al. (1996) Flow-induced vasodilation of the human brachial artery is impaired in patients $<40$ years of age with coronary artery disease. Am J Cardiol 78, 1210-1214.

7. Anderson TJ, Uehata A, Gerhard MD, et al. (1995) Close relation of endothelial function in the human coronary and peripheral circulations. J Am Coll Cardiol 26, 1235-1241.

8. Hollman PCH \& Arts ICW (2000) Flavonols, flavones and flavanols - nature, occurrence and dietary burden. J Sci Food Agric 80, 1081-1093.

9. Tomas-Barberen FA \& Clifford MN (2000) Flavanones, chalcones and dihydrochalcones - nature, occurrence and dietary burden. J Sci Food Agric 80, 1073-1080.
10. de Nigris F, Williams-Ignarro S, Sica V, et al. (2007) Effects of a pomegranate fruit extract rich in punicalagin on oxidationsensitive genes and eNOS activity at sites of perturbed shear stress and atherogenesis. Cardiovas Res 73, 414-423.

11. Edirisinghe I, Banaszewski K, Cappozzo J, et al. (2011) Effect of black currant anthocyanins on the activation of endothelial nitric oxide synthase (eNOS) in vitro in human endothelial cells. J Agric Food Chem 59, 8616-8624.

12. Elies J, Cuinas A, Garcia-Morales V, et al. (2011) Transresveratrol simultaneously increases cytoplasmic $\mathrm{Ca}(2+)$ levels and nitric oxide release in human endothelial cells. Mol Nutr Food Res 55, 1237-1248.

13. Nicholson SK, Tucker GA \& Brameld JM (2010) Physiological concentrations of dietary polyphenols regulate vascular endothelial cell expression of genes important in cardiovascular health. BrJ Nutr 103, 1398-1403.

14. Schroeter H, Heiss C, Balzer J, et al. (2006) (-)-Epicatechin mediates beneficial effects of flavanol-rich cocoa on vascular function in humans. Proc Natl Acad Sci U S A 103, 1024-1029.

15. Gomez-Guzman M, Jimenez R, Sanchez M, et al. (2012) Epicatechin lowers blood pressure, restores endothelial function, and decreases oxidative stress and endothelin-1 and NADPH oxidase activity in DOCA-salt hypertension. Free Rad Biol Med 52, 70-79.

16. Poreba R, Poreba M, Jedrychowska I, et al. (2009) Drinking of chokeberry juice from the ecological farm Dzieciołowo and distensibility of brachial artery in men with mild hypercholesterolemia. Ann Agric Environ Med 16, 305-308.

17. Morand C, Dubray C, Milenkovic D, et al. (2011) Hesperidin contributes to the vascular protective effects of orange juice: a randomized crossover study in healthy volunteers. $A m \mathrm{~J}$ Clin Nutr 93, 73-80.

18. McCall DO, McGartland CP, McKinley MC, et al. (2009) Dietary intake of fruits and vegetables improves microvascular function in hypertensive subjects in a dose-dependent manner. Circulation 119, 2153-2160.

19. Enseleit F, Sudano I, Périat D, et al. (2012) Effects of pycnogenol on endothelial function in patients with stable coronary artery disease: a double-blind, randomized, placebo-controlled, cross-over study. Eur Heart J 33, 1589-1597.

20. Zhu Y, Xia M, Yang Y, et al. (2011) Purified anthocyanin supplementation improves endothelial function via NO-cGMP activation in hypercholesterolemic individuals. Clin Chem 57, 1524-1533.

21. Bondonno C, Yang X, Croft K, et al. (2012) Flavonoid-rich apples and nitrate-rich spinach augment nitric oxide status and improve endothelial function in healthy men and women: a randomized controlled trial. Free Rad Biol Med 51, 95-102.

22. Lapointe A, Couillard C \& Lemieux S (2006) Effects of dietary factors on oxidation of low-density lipoprotein particles. J Nutr Biochem 17, 645-658.

23. Jeong YJ, Choi YJ, Kwon HM, et al. (2005) Differential inhibition of oxidized LDL-induced apoptosis in human endothelial cells treated with different flavonoids. Br J Nutr 93, $581-591$.

24. Safari MR \& Sheikh N (2003) Effects of flavonoids on the susceptibility of low-density lipoprotein to oxidative modification. Prostaglandins Leukot Essent Fatty Acids 69, 73-77.

25. Heinonen M, Rein D, Satue-Gracia MT, et al. (1998) Effect of protein on the antioxidant activity of phenolic compounds in a lecithin-liposome oxidation system. J Agric Food Chem 46, 917-922. 
26. Manach C, Scalbert A, Morand C, et al. (2004) Polyphenols: food sources and bioavailability. Am J Clin Nutr 79, $727-747$.

27. Manach C, Williamson G, Morand C, et al. (2005) Bioavailability and bioefficacy of polyphenols in humans. I. Review of 97 bioavailability studies. Am J Clin Nutr 81, 230S-242S.

28. Hobbs DA, Kaffa N, George TW, et al. (2012) Blood pressure-lowering effects of beetroot juice and novel beetroot-enriched bread products in normotensive male subjects. Br J Nutr 1, 1-9.

29. Chuang SC, Norat T, Murphy N, et al. (2012) Fiber intake and total and cause-specific mortality in the European Prospective Investigation into Cancer and Nutrition cohort. $\mathrm{Am} \mathrm{J}$ Clin Nutr 96, 164-174.

30. Aviram M, Dornfeld L, Rosenblat M, et al. (2000) Pomegranate juice consumption reduces oxidative stress, atherogenic modifications to LDL, and platelet aggregation: studies in humans and in atherosclerotic apolipoprotein E-deficient mice. Am J Clin Nutr 71, 1062-1076.

31. Kris-Etherton PM, Hecker KD, Bonanome A, et al. (2002) Bioactive compounds in foods: their role in the prevention of cardiovascular disease and cancer. Am J Med 113, 71-88.

32. May JM (2000) How does ascorbic acid prevent endothelial dysfunction? Free Rad Biol Med 28, 1421-1429.

33. Ruxton CHS, Gardner EJ \& Walker D (2006) Can pure fruit and vegetable juices protect against cancer and cardiovascular disease too? A review of the evidence. Int $J$ Food Sci Nutr 57, 249-272.

34. Bates B, Lennox A, Swan G. (2010) National Diet and Nutrition Survey: headline results from year 1 of the Rolling Programme (2008/09). http://www.food.gov.uk/ science/dietarysurveys/ndnsdocuments/ndns0809year0801

35. DEFRA (2008) Family Food in 2006. London: The Stationery Office.

36. Markakis P (editor) (1982) Anthocyanins as Food Additives. New York/London: Academic Press.

37. Singleton VL, Orthofer R \& Lamuela-Raventos RM (1999) Analysis of total phenols and other oxidation substrates and antioxidants by means of Folin-Ciocalteu reagent. Meth Enzymol 299, 152-178.

38. Garcia-Macias P, Ordidge M, Vysini E, et al. (2007) Changes in the flavonoid and phenolic acid contents and antioxidant activity of red leaf lettuce (Lollo Rosso) due to cultivation under plastic films varying in ultraviolet transparency. J Agric Food Chem 55, 10168-10172.

39. Ou BX, Hampsch-Woodill M \& Prior RL (2001) Development and validation of an improved oxygen radical absorbance capacity assay using fluorescein as the fluorescent probe. J Agric Food Chem 49, 4619-4626.

40. Liau LS, Lee BL, New AL, et al. (1993) Determination of plasma ascorbic-acid by high-performance liquid-chromatography with ultraviolet and electrochemical detection. J Chromatogr Biomed Appl 612, 63-70.

41. Ross MA (1994) Determination of ascorbic-acid and uric-acid in plasma by high-performance liquid-chromatography. J Chromatogr B: Biomed Appl 657, 197-200.

42. Green LC, Wagner DA, Glogowski J, et al. (1982) Analysis of nitrate, nitrite, and [N-15] labeled nitrate in biological-fluids. Anal Biochem 126, 131-138.

43. Benzie IFF \& Strain JJ (1999) Ferric reducing antioxidant power assay: direct measure of total antioxidant activity of biological fluids and modified version for simultaneous measurement of total antioxidant power and ascorbic acid concentration. Meth Enzymol 299, 15-27.
44. Bub A, Watzl B, Abrahamse L, et al. (2000) Moderate intervention with carotenoid-rich vegetable products reduces lipid peroxidation in men. J Nutr 130, 2200-2206.

45. Leigh-Firbank EC, Minihane AM, Leake DS, et al. (2002) Eicosapentaenoic acid and docosahexaenoic acid from fish oils: differential associations with lipid responses. Br J Nutr 87, 435-445.

46. Esterbauer H, Striegl G, Puhl H, et al. (1989) Continuous monitoring of in vitro oxidation of human low-density lipoprotein. Free Rad Res Commun 6, 67-75.

47. Zhang K \& Zuo YG (2004) GC-MS determination of flavonoids and phenolic and benzoic acids in human plasma after consumption of cranberry juice. J Agric Food Chem 52, 222-227.

48. Rechner AR, Kuhnle G, Bremner P, et al. (2002) The metabolic fate of dietary polyphenols in humans. Free Rad Biol Med 33, 220-235.

49. Mohsen MAE, Marks J, Kuhnle G, et al. (2006) Absorption, tissue distribution and excretion of pelargonidin and its metabolites following oral administration to rats. Br J Nutr 95, 51-58

50. Felgines C, Talavera S, Gonthier MP, et al. (2003) Strawberry anthocyanins are recovered in urine as glucuro- and sulfoconjugates in humans. $J$ Nutr 133, 1296-1301.

51. Kay CD, Mazza G, Holub BJ, et al. (2004) Anthocyanin metabolites in human urine and serum. Br J Nutr 91, 933-942.

52. Hodgson JM, Puddey IB, Croft KD, et al. (2000) Acute effects of ingestion of black and green tea on lipoprotein oxidation. Am J Clin Nutr 71, 1103-1107.

53. Brown MJ, Ferruzzi MG, Nguyen ML, et al. (2004) Carotenoid bioavailability is higher from salads ingested with full-fat than with fat-reduced salad dressings as measured with electrochemical detection. Am J Clin Nutr 80, 396-403.

54. Lotito SB \& Frei B (2004) The increase in human plasma antioxidant capacity after apple consumption is due to the metabolic effect of fructose on urate, not apple-derived antioxidant flavonoids. Free Rad Biol Med 37, 251-258.

55. Caccetta RAA, Croft KD, Beilin LJ, et al. (2000) Ingestion of red wine significantly increases plasma phenolic acid concentrations but does not acutely affect ex vivo lipoprotein oxidizability. Am J Clin Nutr 71, 67-74.

56. Olthof MR, Hollman PCH, Buijsman M, et al. (2003) Chlorogenic acid, quercetin-3-rutinoside and black tea phenols are extensively metabolized in humans. J Nutr 133, 1806-1814.

57. Valentova K, Stejskal D, Bednar P, et al. (2007) Biosafety, antioxidant status, and metabolites in urine after consumption of dried cranberry juice in healthy women: a pilot double-blind placebo-controlled trial. J Agric Food Chem 55, 3217-3224.

58. Natella F, Nardini M, Belelli F, et al. (2007) Coffee drinking induces incorporation of phenolic acids into LDL and increases the resistance of LDL to ex vivo oxidation in humans. Am J Clin Nutr 86, 604-609.

59. Miyagi Y, Miwa K \& Inoue H (1997) Inhibition of human low-density lipoprotein oxidation by flavonoids in red wine and grape juice. Am J Cardiol 80, 1627-1631.

60. Covas MI, de la Torre K, Farre-Albaladejo M, et al. (2006) Postprandial LDL phenolic content and LDL oxidation are modulated by olive oil phenolic compounds in humans. Free Rad Biol Med 40, 608-616.

61. Wagner DA, Schultz DS, Deen WM, et al. (1983) Metabolic fate of an oral dose of N-15-labeled nitrate in humans effect of diet supplementation with ascorbic acid. Cancer Res 43, 1921-1925. 
62. Vauzour D, Houseman E, George T, et al. (2009) Moderate champagne consumption promotes an acute improvement in acute endothelial-independent vascular function in healthy human volunteers. Br I Nutr $\mathbf{1 0 3}$, 1168-1178.

63. Kuhnle G, Spencer JPE, Schroeter H, et al. (2000) Epicatechin and catechin are O-methylated and glucuronidated in the small intestine. Biochem Biophys Res Commun 277, 507-512.

64. Steffen Y, Gruber C, Schewe T, et al. (2008) Mono-Omethylated flavanols and other flavonoids as inhibitors of endothelial NADPH oxidase. Arch Biochem Biophys 469, 209-219.

65. George TW, Niwat C, Waroonphan S, et al. (2009) Effects of chronic and acute consumption of fruit- and vegetablepuree-based drinks on vasodilation, risk factors for CVD and the response as a result of the eNOS G298T polymorphism. Proc Nutr Soc 68, 148-161.

66. George TW, Paterson E, Niwat C, et al. (2009) Effects of chronic and acute fruit and vegetable puree-based drink consumption on cardiovascular disease risk factors. Acta Hort 841, 201-206. 